\title{
Etude des pratiques d'élevage des moutons Peulh du Niger : le Peulh blanc et le Peulh bicolore
}

\author{
Hassane YAYE ABDOU ${ }^{1,2 *}$, Guiguigbaza-Kossigan DAYO ${ }^{3}$, Moumouni ISSA ${ }^{2}$, \\ Mamman MANI $^{1}$, Ibrahim IDI ${ }^{4}$ et Hamani MARICHATOU ${ }^{2}$ \\ ${ }^{1}$ Institut National de Recherche Agronomique du Niger (INRAN), BP : 429, Niamey-Niger. \\ ${ }^{2}$ Université Abdou Moumouni du Niger (UAM). \\ ${ }^{3}$ Centre International de Recherche-Développement sur l'Elevage en zone Subhumide (CIRDES). \\ ${ }^{4}$ Ministère de l'Agriculture et de l'Elevage, Niger. \\ * Auteur correspondant ; E-mail : hassane.yaye@yahoo.fr; Tel : +22796904111 ou +22791129499.
}

\section{REMERCIEMENTS}

Au terme de ce travail, nous tenons à remercier le Programme de Productivité Agricole en Afrique de l'Ouest (PPAAO), CORAF/WECARD et l'UEMOA pour le financement des travaux d'enquête.

\section{RESUME}

Les ovins nigériens représentent $28,4 \%$ du cheptel national et se rencontrent dans toutes les zones agroécologiques. Ils occupent une place importante dans la vie socioéconomique et culturelle du pays. Dans une dynamique de meilleure connaissance de cette espèce, une enquête transversale a été conduite dans les régions de Maradi, Tahoua, Tillabéry et Dosso afin d'étudier les pratiques d'élevage des moutons Peulh. Des questionnaires ont été administrés sur les pratiques d'élevages. Au total, cent neuf (109) éleveurs ont été choisis au hasard selon leur disponibilité à collaborer. Il ressort de cette étude que le pâturage et l'abreuvement sont fournis respectivement par les parcours naturels et les mares semi-permanentes et puits. Les animaux n'ont pas d'habitat et sont en stabulation libre. Les Peulh, les Touareg et les Haoussa sont les principaux éleveurs qui, dans leur majorité, sont âgés entre 15 et 50 (79,8\%), mariés (94,5\%), et analphabètes $(59,6 \%)$. L'héritage et le don constituent les principales voies d'acquisitions des moutons. Les principales entrées et sorties d'animaux sont respectivement les naissances $(69,75 \%)$ et les ventes $(46,90 \%)$. Les moutons sont souvent conduits seuls et la main d'œuvre est majoritairement familiale (81,5\%). La complémentation, la vaccination et le déparasitage sont pratiqués respectivement par $93,6 \%, 88,1 \%$ et $92,7 \%$ des enquêtés. La reproduction est gérée par le contrôle des chaleurs et la pratique de la castration (70,5\% et $44 \%$ des enquêtés). Cette étude a permis d'avoir des caractéristiques sur l'élevage des moutons Peulh du Niger. Cependant, l'utilisation efficace et durable des ces ressources génétiques ovines passera par une meilleure connaissance à travers leur caractérisation phénotypique et moléculaire.

(C) 2019 International Formulae Group. All rights reserved.

Mots clés : Elevage, Ovins, enquête transversale, Peulh, Niger.

\section{Study of the farming practices of Peulh sheep from Niger: white Fulani Sheep and two-tone Fulani sheep}




\begin{abstract}
Sheep in Niger represent $28.4 \%$ of the national livestock and are found in all agro-ecological zones. Sheep play an important socio-cultural and economic role at local and national level. In a dynamic knowledge of this species and breeding practices, a cross-sectional survey was conducted in the regions of Maradi, Tahoua, Tillabery and Dosso. A total of 109 breeders were randomly chosen according to their availability to collaborate. We found that, animals grazing and watering were respectively provided by natural rangelands, semi-permanent pools and wells. The animals don't have habitat and there were free. Peulh, Touareg and Hausa were the main breeders and the age of the majority was between 15 and 50 (79.8\%). Most of them were married $(94.5 \%)$ and illiterate $(59.6 \%)$. Inheritance and gift were the main way to acquire animals. The main inputs and outputs of animals were respectively births $(69.75 \%)$ and sales (46.90\%). Sheep were often driven alone and the manpower was family-owned $(81.5 \%)$. Complementation, vaccination and deworming were performed by $93.6 \%, 88.1 \%$ and $92.7 \%$ of respondents respectively. Reproduction was managed by heat control and castration practice (70.5\% and $44 \%$ of respondents respectively). The present study permitted to have characteristics on the breeding practices of Peulh sheep of Niger. However, the efficient and sustainable use of these sheep resources will require better knowledge through their phenotypic and molecular characterization.
\end{abstract}

(C) 2019 International Formulae Group. All rights reserved.

Keywords: Livestock, ovine, cross-sectional survey, Peulh, Niger.

\section{INTRODUCTION}

Le Niger est un pays continental situé au cœur du sahel. Avec une superficie de 1 $267000 \mathrm{~km}^{2}$, sa population est estimée à 19865066 habitants avec un taux de croissance démographique de 3,9\% (Niger, 2016). Considéré comme le pilier de l'économie nigérienne, l'élevage joue un rôle important dans la sécurité alimentaire, la lutte contre la pauvreté et la cohésion sociale. Il est pratiqué par près de $87 \%$ de la population active soit comme activité principale ou secondaire après l'agriculture. Il contribue pour près de $11 \%$ à la constitution du PIB national et $35 \%$ du PIB agricole (Niger, 2014). Il constitue la seconde source des recettes d'exportation après les ressources minières et contribue également à hauteur de $22 \%$ de la valeur totale des exportations (Niger, 2014).

Le cheptel du Niger est estimé à près de 40 millions de têtes toutes espèces confondues soit près de 16 millions d'Unités Bétail Tropical en 2014 pour une valeur totale de plus de 3000 milliards de FCFA, ce qui présente le pays comme un grand pays d'élevage à vocation essentiellement pastorale (Niger, 2017).

Les petits ruminants (ovins, caprins) ou espèces à cycle court représentent en 2014 plus de 2/3 des effectifs du cheptel. Les ovins qui occupent le troisième rang en termes d'effectifs au niveau du cheptel sont constitués des moutons à poils (le mouton Peulh blanc ou Bali-Bali, le mouton Peulh bicolore ou Oudah, le mouton Touareg ou Ara-Ara et le mouton Balami) et des moutons à laines (le Koundoum, le Hadine et le Daine Zaila). Ces moutons se rencontrent dans toutes les zones agro écologiques du Niger. Compte tenu de l'intérêt sans cesse croissant qu'accorde la population nigérienne aux ovins, de leur rôle important dans l'économie et vu les défis sanitaires et alimentaires imposés par les changements climatiques, les éleveurs de moutons ont besoin d'appuis en termes d'encadrement technique pour mieux valoriser leur élevage.

Ainsi, c'est pour mieux comprendre et apprécier la contribution des productions ovines dans l'économie locale et nationale et dans les perspectives de développement de 
l'élevage ovin, que le présent travail a été initié avec comme objectif d'étudier les pratiques d'élevages des moutons Peulh du Niger.

\section{MATERIEL ET METHODES}

\section{Zone d'étude}

L'étude a été conduite dans 4 régions administratives du Niger (Dosso, Maradi, Tahoua, Tillabéry) et 4 départements (Figure 1 et Tableau 1). Le choix de ces localités s'expliquait par la présence des éleveurs de moutons Peulh (bicolore et blanc) communément appelés «Oudah» pour le bicolore et «Bali Bali» pour le blanc. Le climat de cette zone est de type soudanien pour la région de Dosso et de type sahélien pour les régions de Maradi, Tahoua et Tillabéry. La zone sahélienne comprise entre l'isohyète $100 \mathrm{~mm}$ et $350 \mathrm{~mm}$ comporte une végétation caractérisée d'une part par une flore arborée composée principalement par des Acacia raddiana, Balanites aegyptiaca, Ziziphus mauritania, Calotropis procera, et d'autre part par un couvert herbacé représenté essentiellement par des espèces telles que Panicum turgidum, Aristida mutabilis, Cenchrus biflorus, Eragrostis tremula et Andropogon gayanus. Au niveau de la zone soudanienne, la végétation est caractérisée par une strate ligneuse renfermant des arbustes et des arbres. Cette strate est composée principalement de Combretum sp., d'Acacia sp., de Borassus aethiopium et de Kigelia africana. La strate herbacée est continue avec une prédominance des graminées vivaces telles que Andropogon gayanus, Vitex doniana, Cymbopogon giganteus (Mahamane et al., 2009).

\section{Matériel animal}

Le matériel animal est constitué de deux variantes du mouton Peulh nigérien : le mouton Peulh blanc (Figure 2) et le mouton Peulh bicolore (Figure 3).

\section{Méthode de collecte des données}

Les données ont été collectées par une enquête de perception auprès des éleveurs de moutons Peulh. L'enquête s'est déroulée de mars à août 2016 dans 4 départements du Niger à savoir : Gaya, Bermo, Abalak et Fillingué respectivement dans les régions de Dosso, Maradi, Tahoua et Tillabéry. Les critères de choix des éleveurs enquêtés étaient les suivants : être chef de ménage ou son suppléant, avoir un troupeau de mouton Peulh, être disponible à répondre aux questions. Au total, cent neuf (109) éleveurs répartis dans les différentes communes des départements, ont été sélectionnés.

Les questionnaires ont été administrés au niveau de chaque élevage sous forme d'interview individuelle. Les questions ont porté sur les aspects socio-économiques des éleveurs et leurs pratiques de gestion des moutons Peulhs. Dans toutes les communes, les données ont été collectées par les mêmes enquêteurs en langues bien comprises par les deux parties (enquêteur et enquêté).

\section{Analyse statistique des données}

Les données obtenues ont été saisies et analysées à l'aide du logiciel SPSS 20.0. Les variables quantitatives ont été exprimées en moyenne et écart type ou en médiane tandis que pour les variables qualitatives, des fréquences des différentes modalités ont été calculées. Le test de khi-deux a été utilisé pour déterminer les liaisons entre les variables et les groupes ethniques des éleveurs ou entre les variables et les départements d'élevage. Le test de V de Cramer a été utilisé pour évaluer la force de liaison entre les différentes variables. Le seuil de significativité a été fixé à $\mathrm{p}<0,05$. 


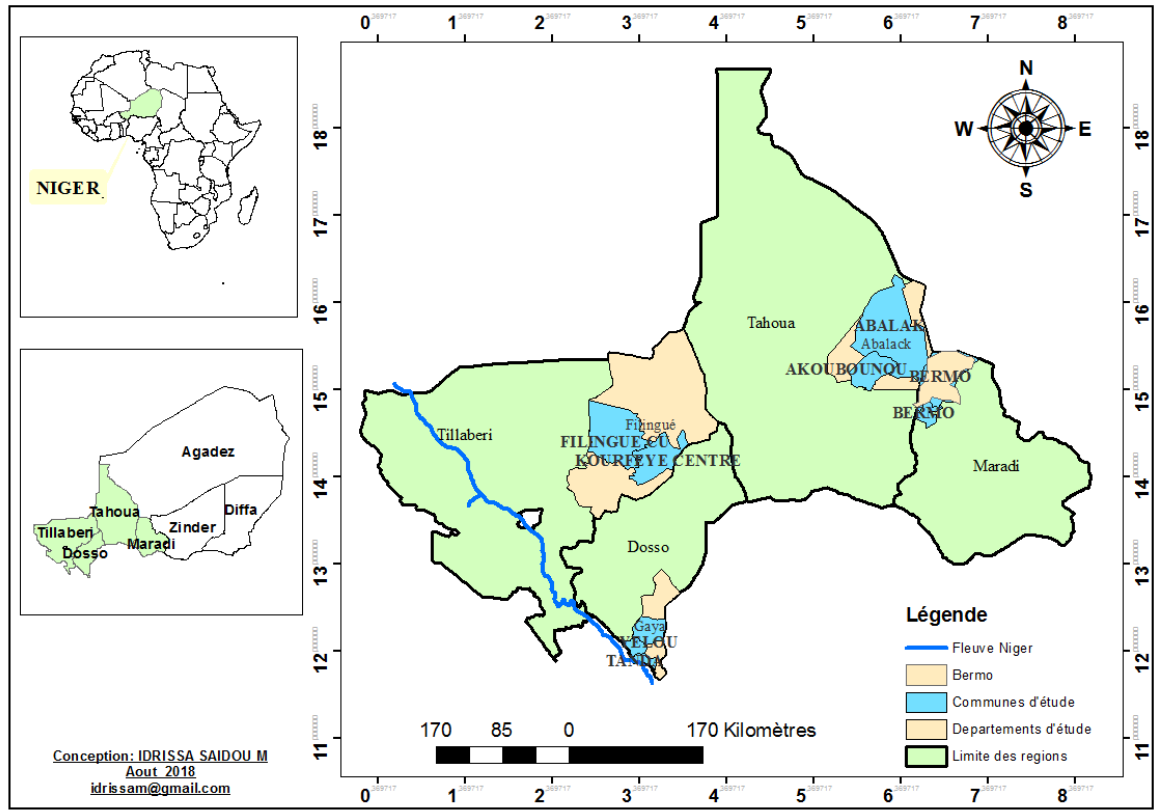

Figure 1 : Zone d'étude.

Tableau 1 : Répartition des enquêtés selon les zones.

\begin{tabular}{lllll}
\hline Régions & Départements & Communes & $\begin{array}{l}\text { Nombres } \\
\text { d'éleveurs }\end{array}$ & Taux/département ou Région (\%) \\
\hline Maradi & Bermo & Bermo & 61 & 55,96 \\
\hline Dosso & Gaya & Tanda & 3 & \multirow{2}{*}{22,01} \\
& & Yellou & 7 & \\
& & Gaya & 14 & 15,59 \\
\hline Tahoua & Abalak & Abalak & 10 & \\
& & Akoubounou & 7 & 6,42 \\
\hline Tillabéry & Fillingué & Fillingué & 4 & \\
& & Chikal (Kourfeye centre) & 3 & $\mathbf{1 0 0}$ \\
\hline Total & & & $\mathbf{1 0 9}$ &
\end{tabular}

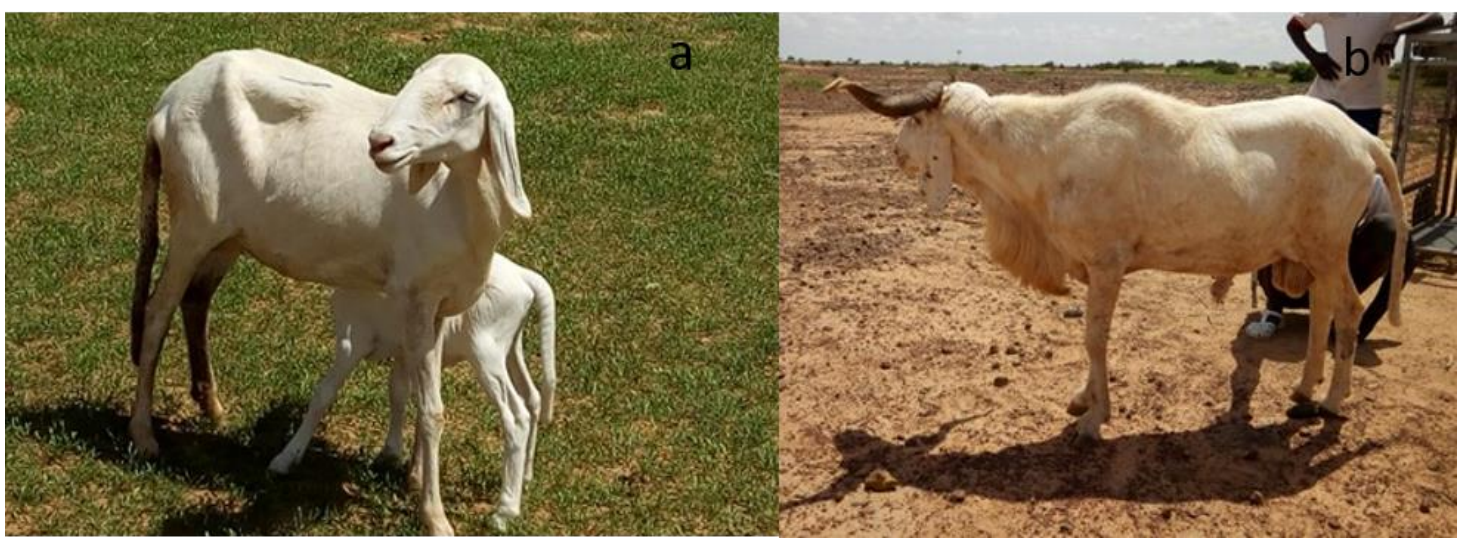

Figure 2 : Mouton Peulh blanc (femelle (a) et mâle (b)). 


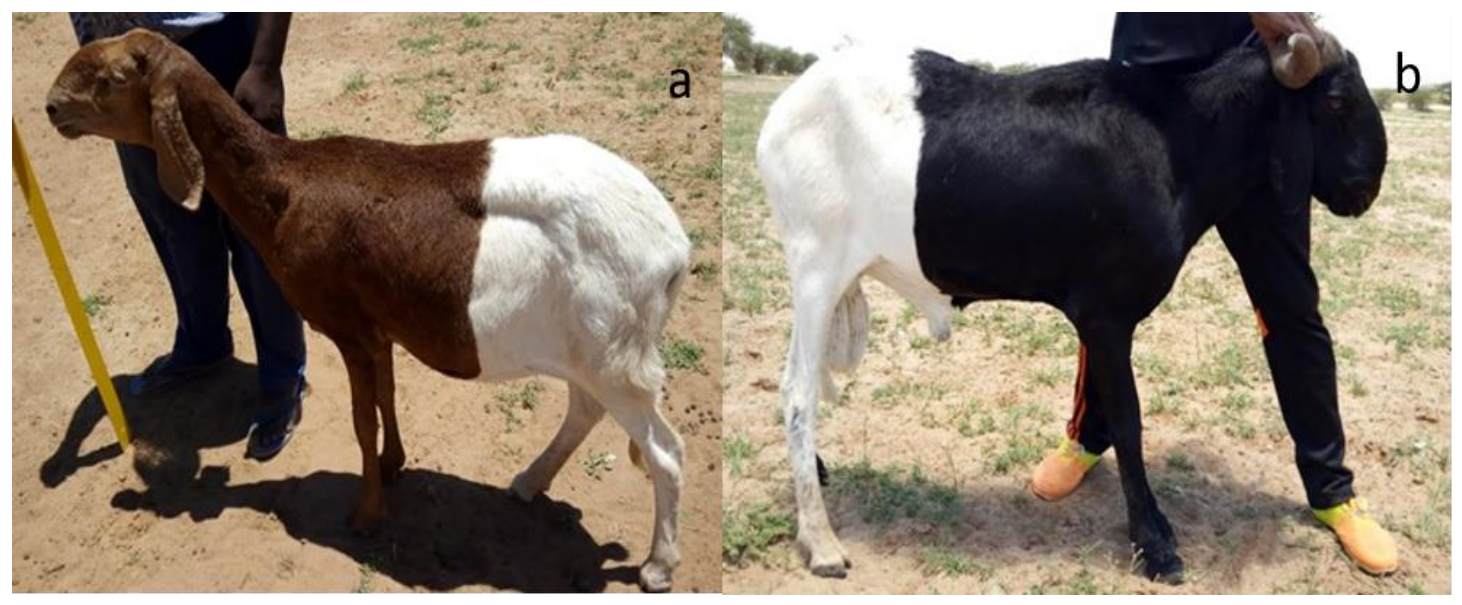

Figure 3 : Mouton Peulh bicolore (femelle (a) et mâle (b)).

\section{RESULTATS \\ Répartition des enquêtés dans la zone d'étude}

Les effectifs des enquêtés par communes et les pourcentages par régions sont consignés dans le tableau 1. Les éleveurs de la région de Maradi étaient les plus représentés avec $55,96 \%$, suivis de ceux de Dosso $22,01 \%$, Tahoua $15,59 \%$ et Tillabéry $6,42 \%$.

\section{Caractéristiques socio-culturelles et économiques des enquêtés}

L'enquête a concerné trois groupes ethnolinguistiques (Tableau 2) sur les neuf (9) que compte le Niger: les Peulh $(78,9 \%)$, les Touareg $(16,5 \%)$ et les Haoussa $(4,6 \%)$. Les caractéristiques sont représentées dans le tableau 3.

La structure familiale des enquêtés, très marquée chez les Peulh, est telle qu'on distingue :

- Un chef d'exploitation qui est le patriarche qui peut détenir encore un troupeau, ils représentent $17,4 \%$ des effectifs enquêtés ;

- Le chef de ménage dépendant qui a son propre troupeau, mais qui est encore sous la responsabilité du chef d'exploitation qu'il peut suppléer, ils représentent $41,3 \%$ des enquêtés ;

- Le chef de ménage indépendant qui a une autonomie de gestion de son troupeau par rapport au chef d'exploitation, ils représentent également $41,3 \%$ des enquêtés.

Les répondants sont tous des hommes (100\%), de confession musulmane, mariés à 94,5\%. Les jeunes et les adultes (de 15 à 50 ans) constituent $79,8 \%$ des enquêtés, pour seulement $20,2 \%$ de personnes âgées de plus de 50 ans. 47,6\% des enquêtés n'ont pas une grande famille en terme de mineurs en charge, au maximum trois (3) mineurs en charge contre $19 \%$ pour les grandes familles qui en comptent plus de six (6) en charge. Les familles monogames sont significativement plus élevées, $(75,2 \%)$ contre $(19,3 \%)$ de polygames et $(5,5 \%)$ de célibataire/divorcé. Le taux des analphabètes (aucune connaissance ni de l'arabe, ni du français) est significativement plus élevé que les alphabétisés, soit 59,6\% des enquêtés contre $28,4 \%$ (alphabétisés arabe) et $11,9 \%$ (alphabétisés français). Les Peulh enquêtés sont analphabètes à $67,4 \%$ contre $38,8 \%$ chez les Touaregs. La principale activité économique des enquêtés est l'élevage, 98,2\% contre $1,8 \%$ pour l'agriculture. La transhumance est le principal mode d'élevage (49,5\%). Les Peulh pratiquent la transhumance à 52,32\% contre $27,77 \%$ chez les Touareg et $80 \%$ des Haoussa. Pendant l'hivernage, les éleveurs quittent les zones de cultures pour les zones pastorales où le pâturage est abondant. 


\section{Structure du cheptel des enquêtés}

Les médianes par espèce et par exploitation dans les différentes régions et ethnies sont présentées dans le Tableau 4. Les bovins, les ovins, les caprins, les asins, les équins et les camelins sont les différentes espèces animales élevées par les enquêtés. Quel que soit le niveau de répartition (région et ethnie), il y a une grande disparité de répartition des animaux de même espèce chez les éleveurs. Les ovins sont numériquement les plus importants par ménage dans toutes les régions et au niveau de toutes les ethnies. Il faut noter que dans toutes les exploitations enquêtées, on ne trouve pas un troupeau entièrement homogène pour l'une des variantes : peulh blanc ou peulh bicolore. Tous les éleveurs possèdent les deux types dans des proportions différentes. Le test de kruskal-Wallis a montré qu'il y a une différence significative entre les départements pour l'effectif moyen par ménage chez les bovins, les ovins, les asins. Les éleveurs de Filingué ont par ménage plus de bovins (51 têtes en moyenne) et d'ovins (148 têtes) que ceux des autres départements. Pour les asins, ce sont ceux de Bermo et Abalak qui sont plus dotés, sept (7 têtes) contre cinq (5) à Filingué et deux (2) à Gaya. En considérant les ethnies des éleveurs, il y a une différence significative seulement pour les effectifs des ovins et des caprins. Ainsi, les enquêtés haoussa ont par ménage plus d'ovins que les autres ethnies, 144 têtes en moyenne contre 59 pour les Peulh.

\section{Mode d'acquisition du troupeau de base chez les enquêtés}

Dans toutes les régions, le troupeau de mouton Peulh d'un ménage appartient à tous les membres : le chef de ménage ou d'exploitation, les femmes et les hommes adultes et les enfants. Chaque composante du ménage peut acquérir ses animaux par plusieurs sources. Les principales voies d'acquisition des moutons Peulh (Figure 4) sont entre autres l'héritage chez les chefs d'exploitation $(78,8 \%)$ et les femmes adultes $(65,3 \%)$. Par contre, le don est le principal mode d'acquisition pour les hommes adultes (75\%) et les enfants $(84,7 \%)$.

\section{Dynamique au sein du troupeau ovin des enquêtés}

Les mouvements d'animaux (entrées et sorties) au niveau des troupeaux ovins selon les enquêtés sur les douze derniers mois sont exprimés en pourcentage (Figure 5).

Les modes d'entrées des troupeaux ovins des exploitations enquêtées étaient constituées des naissances (69,75\%), des achats $(11,49 \%)$, des confiages $(11,49 \%)$, des dons, de la zakat, des dots reçues et des habbanayes. Le habbanaye est un mécanisme traditionnel de solidarité communautaire et d'entraide entre éleveurs. C'est une pratique de la culture Peulh adoptée et adaptée par plusieurs communautés et projets pour lutter contre la pauvreté. Elle consiste à prêter ou à faire un don d'un ou plusieurs bétails pour aider une personne ou un ménage vulnérable et/ou renforcer les liens entre les familles.

Les modes de sorties sont constituées des ventes $(46,90 \%)$, des mortalités $(28,01 \%)$, des abattages $(8,54 \%)$, des habbanayes $(8,40 \%)$, des dons $(4,25 \%)$, des dots, des confiages et la zakat (troisième des cinq piliers de l'Islam est une aumône donnée aux plus pauvres une fois par an).

\section{Pratiques d'élevage des moutons Peulh du Niger selon les ethnies}

Les différentes pratiques d'élevage des moutons Peulh recensées selon les ethnies sont présentées dans le Tableau 5.

\section{Association avec d'autres espèces animales}

Pour toutes les ethnies enquêtées, dans leur majorité, le mouton Peulh est élevé sans être associé à d'autres espèces animales. Les Peulh adoptent cette pratique à 53,57\%, les Touaregs à $44,44 \%$, et les Haoussas à $60 \%$. Pour ceux qui pratiquent l'élevage en association, ils le font avec les bovins, les caprins et autres races ovines.

\section{Main d'ouvre}

La conduite des moutons Peulh et leur surveillance sont faites majoritairement par les membres de la famille pour toutes les 
ethnies sauf chez les Haoussa. Chez ces derniers, $60 \%$ utilisent des bergers salariés.

\section{Habitat des animaux}

Les animaux n'ont pas de logements chez la plupart des éleveurs enquêtés. Ce fait concerne $100 \%$ des Haoussa, $87,5 \%$ des Touareg et $93,97 \%$ des Peulh. Les éleveurs Peulh et Touareg qui possèdent des logements pour leurs animaux les font en enclos de branchage où les moutons sont laissés en stabulation libre.

\section{Alimentation}

L'alimentation est basée principalement sur la conduite des animaux au pâturage sur les parcours naturels. Ainsi, sous la responsabilité du berger, les animaux sont conduits toute la journée vers des zones de pâturage naturel et le soir il les ramène au village ou au campement. Ce mode de conduite est adopté chez $87,5 \%$ des Touareg et $53,01 \%$ des Peulh. Elle est exclusivement de type transhumance chez les Haoussa et le reste des Touareg et des Peulh. Ces éleveurs quittent les zones de cultures, surtout pendant l'hivernage, pour les zones pastorales, et ne retournent que seulement après les récoltes, quand les champs seront libérés.

La complémentation est pratiquée à $100 \%$ chez les Touareg et les Haoussa, à 91,86\% chez les Peulh. Les principaux produits utilisés périodiquement comme compléments par la plupart des éleveurs sont le tourteau de coton $30,39 \%$, les graines de coton $40,08 \%$, les céréales $25,49 \%$, les sons des céréales $78,43 \%$, le sel de cuisine $25,49 \%$, les pierres à lécher locales $86,28 \%$, les fanes de niébé $39,22 \%$ et le foin $22,55 \%$. Pour pallier au déficit fourrager de la saison sèche, la constitution des réserves est faite au niveau de tous les groupes ethniques. Elle concerne $40 \%$ des Haoussa, $66,67 \%$ des Touareg et $56,47 \%$ des Peulh.

\section{Abreuvement}

L'abreuvement en saison des pluies est assuré au niveau de mares semi permanentes $(97,2 \%)$. Par contre, les puits $(82,6 \%)$ et les mares permanentes $(10,10 \%)$ constituent les sources d'abreuvement pour les moutons du Niger en saison sèche. Pendant l'hivernage, la fréquence d'abreuvement est d'une fois le matin avant le départ au pâturage $(26,6 \%)$, ou deux fois, le matin avant le départ et le soir au retour du pâturage $(21,1 \%)$, ou à n'importe quel moment de la journée $(25,7 \%)$ dès lors que l'eau est disponible.

En saison sèche chaude, les abreuvements se font en grande partie une fois le matin avant le départ au pâturage $(58,7 \%)$, une fois tous les deux jours $(23,9 \%)$ et une fois le soir au retour du pâturage $(15,6 \%)$.

En saison sèche froide, les abreuvements se font une fois le matin avant le départ au pâturage $(55,0 \%)$, deux fois le matin avant le départ et le soir au retour du pâturage $(9,2 \%)$, une fois tous les deux jours $(18,3 \%)$.

\section{Suivi sanitaire des animaux}

En matière de la gestion du sanitaire des moutons Peulh, toutes les ethnies font la prévention par la vaccination notamment contre la peste des petits ruminants (PPR), la péripneumonie contagieuse bovine (PPCB). Ils font également le déparasitage interne et externe des animaux. Les principales maladies animales rencontrées dans la zone d'étude étaient le charbon bactéridien, la clavelée, la fièvre aphteuse et les maladies parasitaires.

\section{Gestion de la reproduction}

Le test du khi carré a montré qu'il n'y a pas de différence significative entre la proportion de ceux qui pratiquent le contrôle de chaleur et celle des éleveurs qui ne le font pas. Les principaux signes qui montrent qu'une femelle est en chaleur selon les enquêtés sont: chevauchement, acceptation du mâle, bêlement, et remuement de la queue. Le test du khi carré a montré aussi qu'il n'y a pas de différence significative entre la proportion de ceux qui font le contrôle de la reproduction par la castration et celle des éleveurs qui ne l'ont pas adopté. Les béliers castrés sont exclusivement ceux d'une race autre que le mouton Peulh ou encore ceux qui ont une robe non désirée. Sur les 83 éleveurs ayant répondu à nos questions, 82 renouvèlent les géniteurs par des mâles nés dans le troupeau. Au cas où il y a plusieurs géniteurs, ils sont lâchés libres dans le troupeau quelle que soit l'ethnie ou le département. Le prêt de géniteurs entre éleveurs est également pratiqué dans $92,9 \%$ des cas. 
Tableau 2 : Répartition des enquêtés en fonction de l'ethnie et du département.

\begin{tabular}{llllll}
\hline Départements & & Ethnies & \multicolumn{2}{c}{ Total } \\
\cline { 3 - 5 } & & Haoussa & Touareg & Peulh & \\
\hline Bermo & Effectif & 1 & 14 & 46 & 61 \\
& Freq (\%) & 0,9 & 12,8 & 42,2 & 56,0 \\
\hline Abalak & Effectif & 4 & 2 & 11 & 17 \\
& Freq (\%) & 3,7 & 1,8 & 10,1 & 15,6 \\
\hline Gaya & Effectif & 0 & 0 & 24 & 24 \\
& Freq (\%) & 0,0 & 0,0 & 22,0 & 22,0 \\
\hline Fillingué & Effectif & 0 & 2 & 5 & 7 \\
& Freq (\%) & 0,0 & 1,8 & 4,6 & 6,4 \\
\hline Total & Effectif & 5 & 18 & 86 & 109 \\
& Freq (\%) & 4,6 & 16,5 & 78,9 & 100,0 \\
\hline
\end{tabular}

Tableau 3 : Caractéristiques socio-culturelles des enquêtés.

\begin{tabular}{|c|c|c|c|c|c|c|c|c|}
\hline \multirow[b]{2}{*}{ Variables } & \multirow[b]{2}{*}{ modalités } & \multicolumn{3}{|c|}{ Effectif Ethnie } & \multirow[b]{2}{*}{ Total } & \multirow{2}{*}{$\begin{array}{l}\text { Fréquence } \\
(\%)\end{array}$} & \multirow{2}{*}{$\begin{array}{l}\text { Khi- } \\
\text { deux de } \\
\text { Pearson }\end{array}$} & \multirow{2}{*}{$\begin{array}{l}\text { V de } \\
\text { Cramer }\end{array}$} \\
\hline & & Haoussa & Touareg & Peulh & & & & \\
\hline \multirow{3}{*}{$\begin{array}{l}\text { Tranche } \\
\text { d'âge }\end{array}$} & Jeune $\left[\begin{array}{lll}15 & 35\end{array}\right]$ & 3 & 9 & 38 & 50 & 45,9 & \multirow{3}{*}{0,468} & \multirow{3}{*}{0,128} \\
\hline & Adulte [36 50] & 1 & 8 & 28 & 37 & 33,9 & & \\
\hline & Personne âgée [51 +] & 1 & 1 & 20 & 22 & 20,2 & & \\
\hline \multirow{3}{*}{$\begin{array}{l}\text { Situation } \\
\text { matrimoniale }\end{array}$} & Célibataire/Divorcé & 0 & 4 & 2 & 6 & 5,5 & \multirow{3}{*}{0,011} & \multirow{3}{*}{0,244} \\
\hline & Polygame & 2 & 3 & 16 & 21 & 19,3 & & \\
\hline & Monogame & 3 & 11 & 68 & 82 & 75,2 & & \\
\hline \multirow{3}{*}{$\begin{array}{l}\text { Niveau } \\
\text { d'instruction }\end{array}$} & Alphabétiser français & 1 & 5 & 7 & 13 & 11,9 & \multirow{3}{*}{0,004} & \multirow{3}{*}{0,267} \\
\hline & Alphabétiser Arabe & 4 & 6 & 21 & 31 & 28,4 & & \\
\hline & Analphabète & 0 & 7 & 58 & 65 & 59,6 & & \\
\hline \multirow{3}{*}{$\begin{array}{l}\text { Taille de la } \\
\text { famille }\end{array}$} & $\begin{array}{l}\text { Petite famille (0à3 } \\
\text { mineurs en charge) }\end{array}$ & 3 & 10 & 37 & 50 & 47,6 & \multirow{3}{*}{0,893} & \multirow{3}{*}{0,073} \\
\hline & $\begin{array}{l}\text { Moyenne famille (4à6 } \\
\text { mineurs en charge) }\end{array}$ & 1 & 5 & 29 & 35 & 33,3 & & \\
\hline & $\begin{array}{l}\text { Grande famille ( }+6 \\
\text { mineurs en charge) }\end{array}$ & 1 & 3 & 16 & 20 & 19,0 & & \\
\hline \multirow[b]{2}{*}{$\begin{array}{l}\text { Mode } \\
\text { d'élevage }\end{array}$} & Transhumant & 4 & 5 & 45 & 54 & 49,5 & \multirow[b]{2}{*}{0,126} & \multirow[b]{2}{*}{0,182} \\
\hline & Nomade & 0 & 8 & 19 & 27 & 24,8 & & \\
\hline
\end{tabular}


$\begin{array}{llllll}\text { Sédentaire } & 1 & 5 & 22 & 28 & 25,7\end{array}$

\begin{tabular}{|c|c|c|c|c|c|c|c|c|}
\hline \multirow{2}{*}{$\begin{array}{l}\text { Principale } \\
\text { activité de } \\
\text { l'exploitation }\end{array}$} & Agriculture & 1 & 0 & 1 & 2 & 1,8 & \multirow[b]{2}{*}{0,008} & \multirow[b]{2}{*}{0,298} \\
\hline & Elevage & 4 & 18 & 85 & 107 & 98,2 & & \\
\hline \multirow{3}{*}{$\begin{array}{l}\text { Niveau } \\
\text { décision }\end{array}$} & Chef d'exploitation & 1 & 3 & 15 & 19 & 17,4 & \multirow{3}{*}{0,879} & \multirow{3}{*}{0,074} \\
\hline & $\begin{array}{l}\text { Chef de ménage } \\
\text { indépendant }\end{array}$ & 1 & 7 & 37 & 45 & 41,3 & & \\
\hline & $\begin{array}{l}\text { Chef de ménage } \\
\text { dépendant (suppléant) }\end{array}$ & 3 & 8 & 34 & 45 & 41,3 & & \\
\hline \multirow{3}{*}{$\begin{array}{l}\text { Croyance } \\
\text { religieuse }\end{array}$} & Musulman & 5 & 18 & 86 & 109 & 100,0 & & \\
\hline & Chrétien & 0 & 0 & 0 & 0 & 0,0 & & \\
\hline & Autres & 0 & 0 & 0 & 0 & 0,0 & & \\
\hline
\end{tabular}

Tableau 4 : Structure du cheptel.

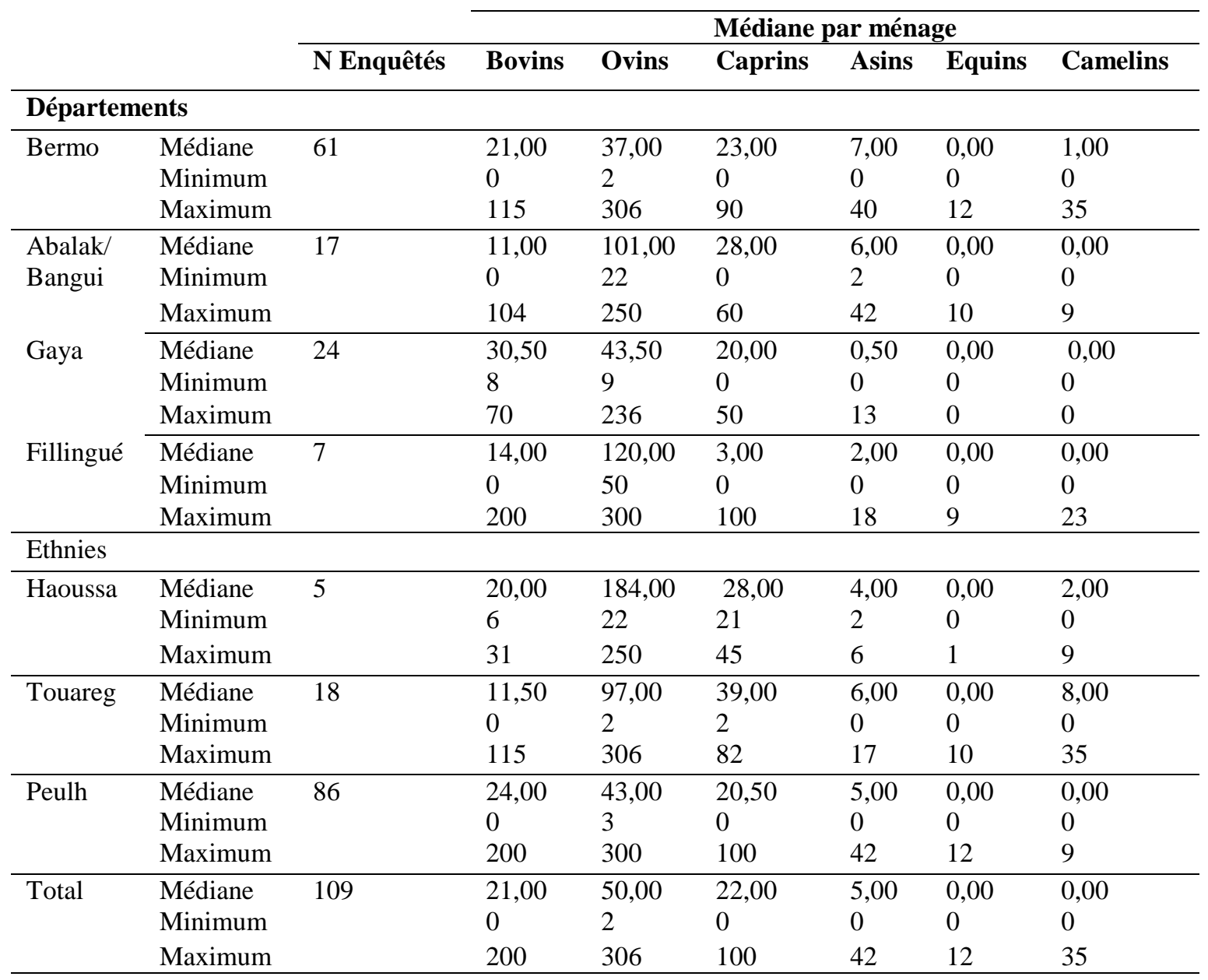


Tableau 5 : Les pratiques d'élevage des moutons Peulh du Niger selon les ethnies.

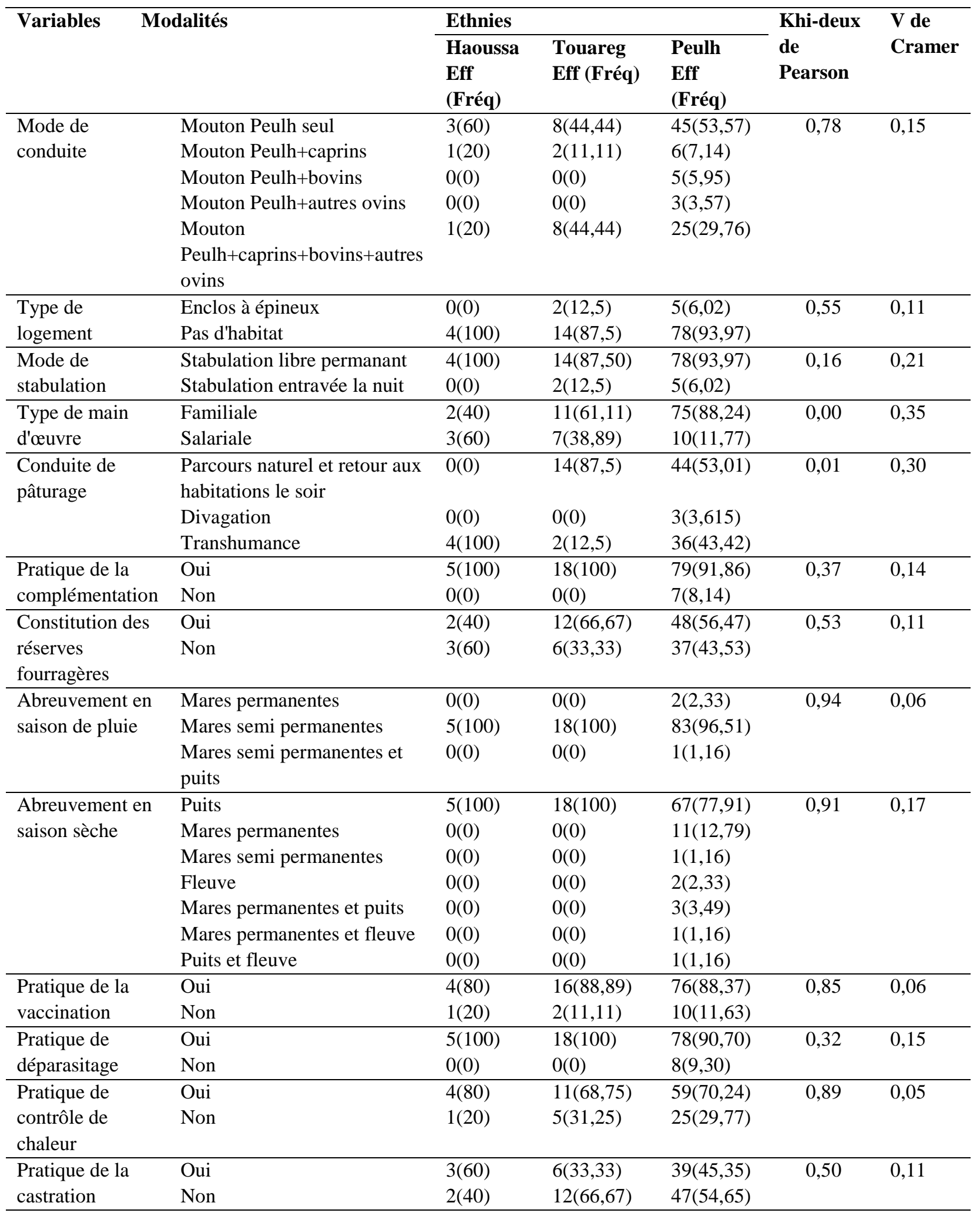




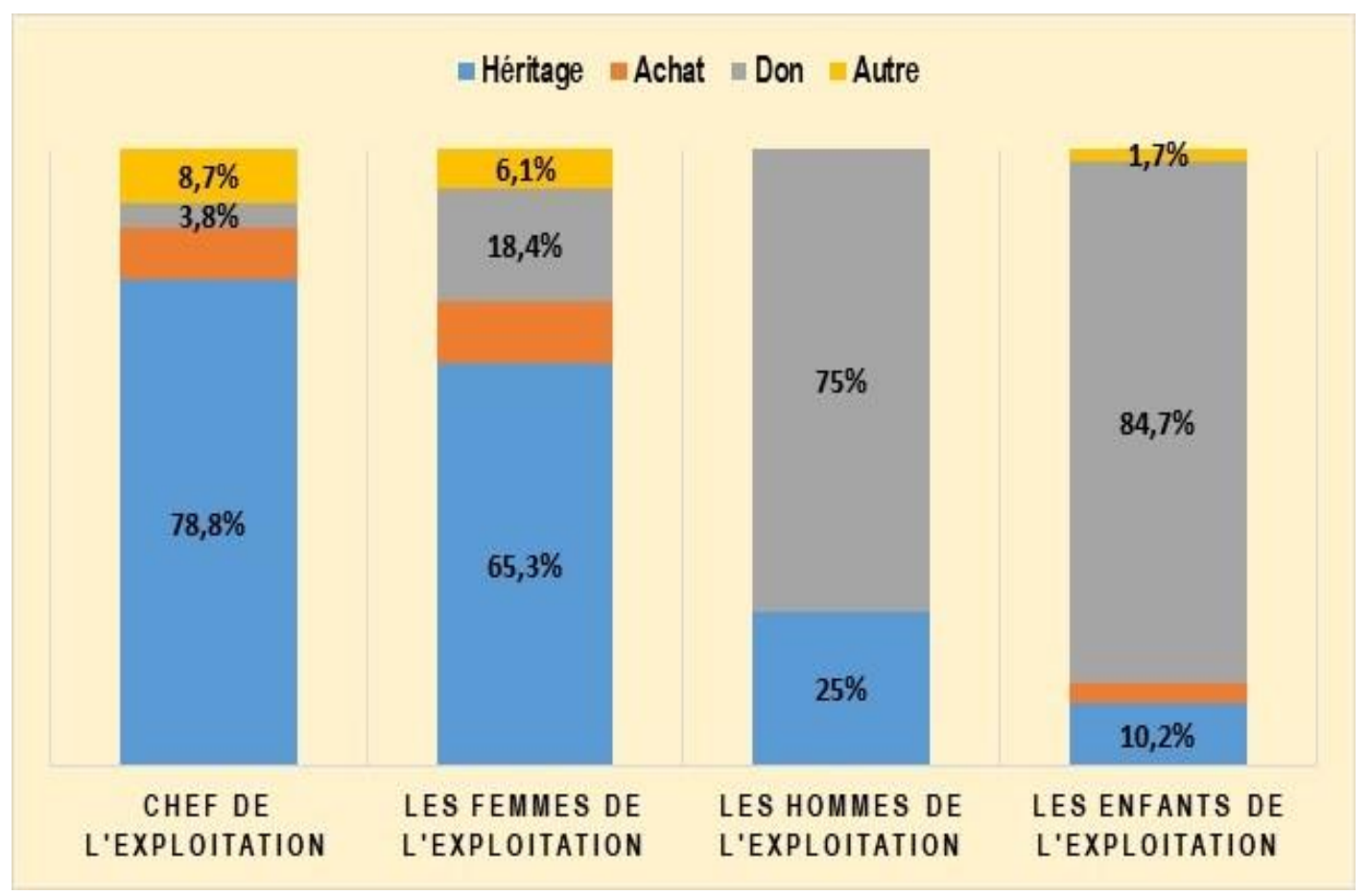

Figure 4 : Mode d'acquisition des moutons Peulh.

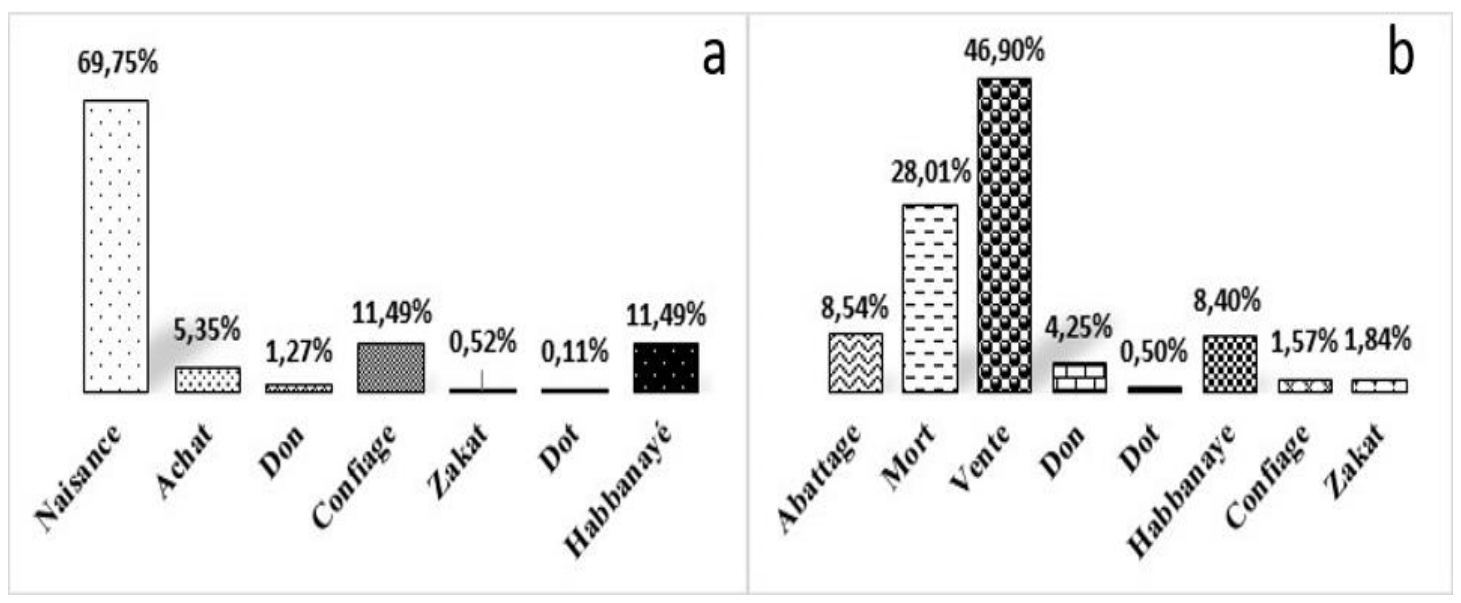

Figure 5 : Les entrées (a) et sorties (b) des animaux au niveau de l'exploitation.

\section{DISCUSSION}

L'enquête de perception sur les caractéristiques de l'élevage des moutons Peulh auprès des éleveurs nous a permis de comprendre les pratiques d'élevage auxquelles sont soumis ces ovins. Les régions enquêtées à savoir Maradi, Dosso, Tahoua et Tillabéry sont les grandes zones d'élevage connues des moutons Peulh et constituent à elles seules $60 \%$ du cheptel nigérien toutes espèces confondues et $61,43 \%$ du cheptel ovin (Niger, 2014).

Le nombre élevé des enquêtés dans la région de Maradi s'explique par le grand nombre d'éleveurs du mouton peulh dans la région et par la facilité d'accès à l'information dans la zone pendant l'étude. Les groupes ethnolinguistiques majoritairement rencontrés 
dans cette étude, les Peulh, les Touareg et les Haussa sont traditionnellement les principaux éleveurs pasteurs des moutons Peulh au Niger.

La présente étude a montré que la constitution des troupeaux de base se fait principalement de deux manières : l'héritage chez les femmes et les chefs de l'exploitation et le don chez les hommes et les enfants de l'exploitation. Ces résultats sont similaires à ceux rapportés par Dicko et al. (2006) qui avaient montré que le bétail est souvent acquis via l'héritage, l'achat, le don.

L'étude a montré que l'élevage est pratiqué aussi bien par les jeunes que les personnes âgées mais, seuls les hommes étaient des chefs d'exploitation ou chef de ménage. Le fort taux des mariés chez les enquêtés traduit le niveau de responsabilité sociale et l'expérience des éleveurs dans la gestion et la conduite des animaux. Par contre, les jeunes (45,9\% des enquêtés) jouent un rôle important dans le transfert des connaissances d'une génération à l'autre. Le capital expérience des éleveurs compenserait l'effet de leur analphabétisme et de leur faible niveau d'instruction comme cela a été aussi observé dans l'étude de Mani (2013) contrairement aux éleveurs urbains qui leur grande majorité étaient scolarisés (Lawal et al., 2018; Sanon et al., 2018). L'étude a montré un fort pourcentage des petites et moyennes familles (plus de 80,9\% des personnes enquêtées) par rapport aux familles de grande taille ainsi que la proportion élevée des chefs de ménage indépendant et des chefs de ménage dépendant $(82,6 \%)$ dans la prise des décisions. Cette observation témoigne de la forte implication des jeunes et des adultes qui se déplacent de façon saisonnière et cyclique avec les animaux à la quête perpétuelle de l'eau et des pâturages sur des parcours naturels et du retrait progressif des personnes âgées. Ces personnes âgées, considérées comme des patriarches, sont généralement les chefs d'exploitation tandis que la responsabilité de la gestion du bétail revient au chef de famille.

La forte implication des jeunes dans l'élevage des moutons Peulh observée dans notre étude est contraire aux résultats de
Hamadou et al. (2015) qui avait rapporté pour le cas de l'élevage du mouton Koundoum du Niger où les jeunes et adultes sont moins impliqués dans la conduite des animaux à cause d'une part de l'exode rural et de l'autre part de l'organisation sociale des ménages dans la vallée du fleuve Niger, ou encore d'une négligence de la race Koundoum.

La présente étude a montré que la mobilité (transhumance ou nomadisme) concerne près de $75 \%$ de l'élevage des moutons peulh. Ces résultats sont similaires à ceux observés au Sénégal sur le mouton Peulpeul par Ndiaye et al. (2018), au Mali par Ham et al. (2011). En effet, la pratique de la transhumance a constitué pendant longtemps pour la majorité des éleveurs sahéliens, une stratégie efficace d'adaptation aux aléas climatiques. La transhumance est considérée également comme un système d'exploitation opportuniste des ressources pastorales face aux crises fourragères saisonnières, en tirant parti de la diversité écologique et de la complémentarité entre les différentes zones agro climatiques du pays (FAO, 2012 ; Kiema et al., 2014). Ce mode d'élevage explique l'absence d'habitat ou de logement pour les animaux révélé dans la présente étude. Ces résultats sont différents de ceux trouvés par Ndiaye et al. (2018) sur l'étude de l'élevage du mouton Peul-peul du Sénégal pour lequel des habitats sont construits mais sont constitués par des enclos faits de branchages d'arbustes épineux et dépourvus de toit.

\section{Structure et effectifs des troupeaux}

Le cheptel des exploitations enquêtées reflète celle du pays dans sa composition avec une prédominance des petits ruminants (ovins et caprins) à hauteur de $88,76 \%$ et conformément à ceux trouvés par Mani (2013) et Niger (2014).

L'effectif moyen (71,75 têtes) de mouton Peulh par troupeau enquêté témoigne de l'importance qu'accordent les éleveurs pour la race ovine Peulh. Cette valeur est supérieure à celle trouvée par Amegee (1983) au Togo avec 7 à 8 brebis, par Thys and Ekembe (1992) au Cameroun avec un effectif moyen de 8,2 têtes, par Ousseini (2011) au 
Sénégal avec un effectif moyen de 14 têtes et par Hounzangbe Adote et al. (2011) au Benin avec 2 à 10 têtes. Ces différences peuvent être expliquées par le fait que dans les systèmes pastoraux purs, la taille du troupeau est évidement plus importante que dans les systèmes agro-pastoraux (Rade, 1994).

Cependant le faible pourcentage observé pendant l'enquête au niveau des asins, des équins et des camelins peut se justifier par le fait que ces derniers sont très rarement utilisés pour couvrir les besoins financiers des éleveurs. Ils interviennent dans le transport des hommes et de leurs bagages et aux corvées quotidiennes, l'exhaure pendant les saisons sèches au niveau des puits qui sont généralement très profonds dans ces zones.

\section{Exploitation des animaux}

Les entrées dans les troupeaux enquêtés sont constituées des facteurs de la constitution et du renouvellement significatif des troupeaux. Les naissances, le habbanaye, le confiage et les achats constituent les principaux modes de constitution des troupeaux avec une part importante pour les naissances (69,75\%). Ces résultats corroborent ceux trouvés par Mani (2013) sur la chèvre du Sahel au Niger.

Quant aux sorties qui constituent les principaux facteurs de la baisse de la taille du troupeau, elles sont représentées par les ventes suivies des mortalités, des abattages, du habbanaye et des dons. Les ventes concernent principalement les mâles. Ce constat a été également rapporté par Traoré et al. (2006) sur la race ovine locale Mossi au Burkina Faso. Dans les différents troupeaux, les effectifs des brebis sont supérieurs à ceux des béliers à cause de la forte exploitation de ces derniers par les ventes et les abattages. Cette observation a été faite par Thys and Ekembe (1992) au Cameroun et par Ousseini (2011) au Sénégal.

Les principaux facteurs d'entrées et de sortie, surtout les naissances et les ventes, sont fonctions de la disponibilité du fourrage, donc d'une bonne saison hivernale. En effet, l'activité sexuelle des moutons n'est à son maximum que quand ils sont bien alimentés
(Boly et al., 1993; Boussena, 2013) et les animaux ne sont rentables par la vente que quand ils sont bien embouchés (Sangaré et al., 2005).

La complémentation telle que définie par Moulin (1993) est pratiquée par presque tous les éleveurs, et même, durant les périodes de disette. Les compléments utilisés sont des productions locales et sont issus des sousproduits agricoles ou agroindustriels $\mathrm{du}$ terroir. Ils sont achetés sur les marchés locaux et/ou proviennent du stock de réserves fourragères des agro-pasteurs.

\section{Conduite au pâturage}

Les moutons Peulh sont le plus souvent conduits sans association avec d'autres races ovines. Cette pratique est favorable pour la pureté ou l'homogénéité de cette race dont les deux variantes sont jalousement gardées par les éleveurs (Dayo et al., 2015). Dans quelques cas, les Peulh, les Haoussa et les Touareg conduisent ensembles les ovins et les caprins. Seuls les Peulhs associent parfois les ovins aux bovins pour la conduite des troupeaux. Ce système de pâture est connu pour favoriser d'une part l'état de santé (en réduisant l'infestation parasitaire des animaux) et les performances des animaux et d'autre part une bonne composition des espèces fourragères du milieu (Hoste et al., 2003; Meisser et al., 2013; D'Alexis et al., 2014). En Afrique de l'Ouest, le type de main d'œuvre utilisée est très variable en fonction des ethnies. En effet, dans la présente étude, la conduite des moutons Peulh est en majeure partie de type familial chez les éleveurs Peulh et Touareg comme pour le mouton Ladoum du Sénégal (Ousseini, 2011) ou la chèvre du Sahel au Niger (Mani, 2013). Ces résultats corroborent ceux trouvés par Lawal et al. (2018) dans les exploitations urbaines et périurbaines de Niamey au Niger. Par contre, chez les éleveurs Haoussa, la conduite du troupeau est en grande partie de type salarial. Les troupeaux de petits ruminants sont confiés soit en gardiennage rémunéré soit en pension chez d'autres exploitants (métayage) au Bénin (Hounzangbe Adote et al., 2011). Au Sénégal, elle est familiale et/ou salariée avec un berger 
pour les moutons Peul-peul (Ndiaye et al., 2018).

Pour le suivi sanitaire des animaux, les différents acteurs (éleveurs, services publics de l'Etat et vétérinaires privés) accordent une attention particulière pour garantir la santé des animaux et de leurs propriétaires pour ce qui concerne les zoonoses. En effet, l'Etat du Niger organise chaque année des campagnes de vaccination gratuites. Ainsi, les produits et services vétérinaires sont disponibles au niveau des pharmacies et cliniques vétérinaires privés ou des agents vétérinaires de l'Etat. L'automédication est pratiquée par presque tous les éleveurs car il existe des marchés incontrôlés de vente de médicaments vétérinaires. Cette pratique n'est pas conforme aux règles d'hygiènes de production et peut favoriser le développement de la résistance des pathogènes aux différentes molécules médicamenteuses, la présence des résidus de médicament dans les produits animaux si les délais d'attente ne sont pas respectés.

La reproduction des animaux est gérée par le contrôle des chaleurs et la pratique de la castration des béliers. Ces pratiques courantes servent à contrôler la reproduction et la sélection des meilleurs animaux du troupeau. Par ces pratiques, presque tous les éleveurs regroupent les naissances suivant des périodes bien déterminées bien que chez le mouton Peulh, la reproduction s'étale sur toute l'année (Yenikoye et al., 1981; Issa et al., 2001; Lurette et al., 2015).

\section{Conclusion}

La présente étude a montré que les moutons Peulh du Niger sont rencontrés dans toutes les zones agro-écologiques du Niger auxquelles ils sont parfaitement adaptés. Le système d'élevage de ces moutons est de type extensif traditionnel basé essentiellement sur l'exploitation des parcours naturels. Ils sont dans la plupart des cas conduits seuls sans association avec une autre espèce et/ou race ovine. La taille des troupeaux est très variable. Sans habitats les troupeaux sont conduits par une main d'œuvre majoritairement familiale. Les mares semi-permanentes et les puits constituent les sources majoritaires pour l'abreuvement.

Les Peulh, les Touareg et les Haoussa sont les principales ethnies qui élèvent cette race ovine. Il ressort également de cette étude que ces éleveurs conduisent de la même manière les troupeaux des deux variantes du mouton peulh du Niger (bicolore/Oudah ou blanc/Bali-Bali). Toutefois, le choix des géniteurs est favorable à l'homogénéité des deux variantes.

Le troupeau de base est souvent constitué par l'héritage et/ou par le don. Les naissances sont les principaux facteurs de la reconstitution des troupeaux ovins et les ventes et les morts sont les facteurs principaux dans l'exploitation des troupeaux. Les différentes pratiques sur l'alimentation (la complémentation), la santé animale (la vaccination, le déparasitage), la reproduction (la castration, le contrôle des chaleurs) sont connues et très pratiquées par les éleveurs dans l'objectif d'un élevage meilleur pour les animaux. Pour maintenir l'homogénéité de ces deux variantes de la race il est nécessaire de conduire des études de caractérisation (phénotypique et moléculaire) et d'appuyer les éleveurs à s'organiser en association de race.

\section{CONFLITS D'INTERETS}

Les auteurs déclarent qu'il n'existe aucun conflit d'intérêts lié à la présente étude.

\section{CONTRIBUTIONS DES AUTEURS}

MI, G-KD et HM ont été les promoteurs de ce travail et ont approuvé la version finale $\mathrm{du}$ présent manuscrit après avoir intégré leurs observations.

Avec les promoteurs, nous avons réalisé la conception et la mise au point du protocole scientifique et des fiches d'enquête. HYA a été l'investigateur principal de ce travail. Il a organisé et supervisé la collecte des données sur le terrain et leur dépouillement. Avec l'aide de MM, il a réalisé le traitement et les analyses statistiques des données. Il a ensuite interprété les résultats et rédigé le manuscrit. II a servi de guide et de facilitateur sur le terrain. 


\section{REMERCIEMENTS}

Nos remerciements vont également à la Coordination nationale du Projet de gestion et de valorisation des ressources génétiques animales et aquacoles dans l'espace UEMOA (PROGEVAL) financé par la Convention entre le CORAF/WECARD et l'UEMOA pour son accompagnement dans l'organisation de la collecte des données.

\section{REFERENCES}

Boly H, Koubaye A, Martinez MV, Yenikoye A. 1993. Gestation et réprise de l'activité sexuelle après le part chez la brebis Djallonké, variété "Mossi". Rev. Elev. Méd. Vét. Pays Trop., 46(4): 631-636 DOI: https://doi.org/10.19182/remvt.9419

Boussena S. 2013. Performances de reproduction chez les ovins Ouled Djellal: Avènement de la puberté et évolution des caractéristiques séminales chez le mâle jusqu'à l'âge de 1 an. Doctorat Es-Sciences, Université Constantine 1, p. 234.

D'Alexis S, Angeon V, Arquet R, Boval M, 2015. Les systèmes mixtes d'élevage de petits ruminants et de bovins: Une alternative pour améliorer les performances animales au pâturage. Innovations Agronomiques, 43: 19-28. HAL Id : hal-01222900

Dayo GK, Alfa E, Talaki E, Soedji K, Sylla S, Dao B. 2015. Caractérisation phénotypique du mouton de Vogan du Togo et relation avec le mouton Djallonké et le mouton sahélien. Animal Genetic Resources, 56: 63-78. DOI: $10.1017 / \mathrm{S} 207863361500003 \mathrm{Xv}$

Dicko MS, Djitèye MA, Sangaré M. 2006. Les systèmes de production animale au Sahel. Sécheresse, 17(1-2): 83-97.

Ham F, Metais T, Hoorelbeke P, Fillol E, Crahay P. 2011. One horn of the cow : an innovative GIS-based surveillance and early warning system pastoral areas of Sahel. ACF, FAO, p. 2.

Hamadou I, Moula N, Siddo S, Marichatou H, Issa M, Leroy $\mathrm{P}$, Antoine-Moussiaux $\mathrm{N}$. 2015. La race de mouton
Koundoum au Niger : étude morphobiométrique et description du système de production. JARTS, 116(1): 49-58. DOI : http://nbnresolving.de/urn:nbn:de:hebis:342015011347146

Hoste H, Guitard J, Pons J. 2003. Pâturage mixte entre ovins et bovins intérêt dans la gestion des strongyloses gastro intestinales. Fourrages, 176, 425-436.

Hounzangbe-Adote MS, Azando E, Awohouedji Y. 2011. Biodiversité dans les zones d'élevage des petits ruminants Mammifères domestiques BENIN. In Atlas de la Biodiversité de l'Afrique de l'Ouest. Brice Sinsin \& Dorothea Kampmann (eds) : $506-518$. https://www.researchgate.net/publication /262564761

Issa M, Yenikoye A, Marichatou H, Banoin M. 2001. Spermogramme de béliers Peuls bicolores et Touaregs : influence du type génétique et de la saison. Rev. Élev. Méd. Vét. Pays Trop., 54(34): 269-275. DOI: https://doi.org/10.19182/remvt.9784

Kiema A, Tontibomma BG, Zampaligré N. 2014. Transhumance et gestion des ressources naturelles au Sahel : contraintes et perspectives face aux mutations des systèmes de productions pastorales. VertigO - la revue électronique en sciences de l'environnement, 14(3). DOI : https://id.erudit.org/iderudit/1034947ar

Lawal AM, Chaibou M, Mani M, Garba M, Gouro A. 2018. Pratiques d'éleveurs et résultats économiques d'élevage dans les exploitations urbaines et périurbaines de Niamey. Int. J. Biol. Chem. Sci., 12: 294309.

DOI: https://dx.doi.org/10.4314/ijbcs.v12i1.24

Lurette A, Fréret S, Chanvallon A, Experton C, Frappat B, Gatien J, Martineau C. 2015. La gestion de la reproduction en élevages ovins et caprins, conventionnels et biologiques: état des lieux, intérêt et acceptabilité de nouveaux outils dans six bassins de production en France. 
INRA Productions Animales, 29(3): 163184.

Mahamane A, Saadou M, Danjimo MB, Saley K, Yacoubou B, Diouf A, Morou B, Mamane Maarouhi I, Soumana I, Tanimoune A. 2009. Biodiversité végétale au Niger: État des connaissances actuelles. Ann. Univ. Lomé (Togo), Séries Sciences Tome, 18: 81-93.

Mani M. 2013. Caractérisation phénotypique et zootechnique de la chèvre du Sahel élevée au Niger. Doctorat Unique, Université Cheikh Anta Diop de Dakar, Université Abdou Moumouni de Niamey, p. 160.

Meisser M, Frey C, Deléglise C, Mosimann E. 2013. Pâturage mixte bovins-ovins en moyenne montagne: trois années d'essai dans le Jura suisse. Fourrages, 216 : 305-311.

Moulin CH. 1993. Le concept de fonctionnement de troupeau : Diversité des pratiques et variabilité des performances animales dans un système agropastoral sahélien. Etudes et Recherches sur les Systèmes Agraires et le Développement, p. 21.

Ndiaye B, Diouf MN, Ciss M, Wane M, Diop M, Sembène M. 2018. Morphologie et pratiques d'élevage du mouton Peul-peul du Sénégal. Inter. J. Adv. Res., 6(5): 727 738. DOI:10.21474/IJAR01/7089

Niger. 2014. Atlas sur l'élevage au Niger : L'élevage au Niger, une richesse sans fin. Ministère de l'élevage, Direction des statistiques, p. 133.

Niger. 2016. Tableau de bord social. Ministère du Plan de l'Aménagement du Territoire et du Développement Communautaire, Institut National de la Statistique du Niger, p. 117.

Niger. 2017. Plan de Développement Economique et Social 2017-2021 : Un Niger renaissant pour un peuple prospère. Ministère du Plan, p. 198.
Ousseini H. 2011. Analyse socioéconomique des élevages du mouton Ladoum dans les communes de Thiès/Sénégal. Master 2 de Productions Animales et Développement Durable Option : Economie et Politiques d'Elevage. Université Cheikh Anta Diop, Sénégal, p. 33.

Rade MC. 1994. Contribution à l'étude des caractéristiques morphologiques et zootechnique des petits ruminants en Afrique tropicale : Synthèse bibliographique. These de Doctorat Vétérinaire, Université Cheikh Anta Diop, senegal, p. 130.

Sangaré M, Thys E, Gouro SA. 2005. L'alimentation des ovins de race locale: Techniques d'embouche ovine, choix de l'animal et durée. Centre international de recherche développement sur l'élevage en zone subhumide, p. 8.

Sanon H, Some S, Obulbiga M, Oubda F, Bamouni I. 2018. Analyse de la structure et $\mathrm{du}$ fonctionnement de la filière fourrage dans les villes de Ouagadougou et Bobo-Dioulasso au Burkina Faso. Int. J. Biol. Chem. Sci., 12: 1247-1259. DOI:

https://dx.doi.org/10.4314/ijbcs.v12i3.14

Thys E, Ekembe T. 1992. Elevage citadin des petits ruminants à Maroua (Cameroun). Cahiers Agric., 1(4): 249-255. http://dspace.itg.be/handle/10390/3939

Traoré A, Tamboura HH, Kaboré A, Yaméogo N, Bayala B, Zaré I. 2006. Caractérisation morphologique des petits ruminants (ovins et caprins) de race locale "Mossi" au Burkina Faso. AGRI, 39: $39-50 . \quad$ DOI: 10.1017/S1014233900002121

Yenikoye A, Andre D, Ravault J, Mariana J. 1981. Etude de quelques caractéristiques dereproduction chez la brebis Peulh, du Niger. Reprod. Nutr. Dévelop., 21(6A): 937-951. DOI: 10.1051/rnd:19810706 